\title{
Iniciação Científica \\ Distribución potencial de Culiseta melanura (Coquillett) (Diptera: Culicidae) en América
}

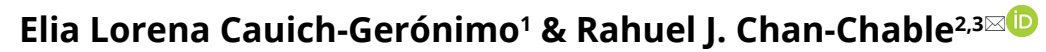

\begin{abstract}
1. Universidad Virtual del Estado de Guanajuato (UVEG), Purísima del Rincón, Guanajuato, México. 2. Universidad Autónoma Agraria Antonio Narro, Unidad Laguna, Torreón, Coahuila, México. 3. Servicios Estatales de Salud de Quintana Roo, Chetumal, Quintana Roo, México.
\end{abstract}

\section{Entomology Beginners, vol. 2: e012 (2021)}

\begin{abstract}
Resumen. Culiseta melanura es un mosquito importante debido a su papel en el ciclo de transmisión del virus de la Encefalitis Equina del Este. En este estudio determinamos la distribución potencial de $C$. melanura en el continente americano y la importancia de las variables bioclimáticas en la conformación de dicha distribución. Para representar la distribución potencial de $C$. melanura se utilizó un modelo de máxima entropía (MaxEnt, versión 3.4.1). Se utilizó un total de 80 registros geográficos obtenidos de GBIF y literatura pertinente. Los datos de clima se obtuvieron de 19 capas climáticas disponibles en la base de datos WorldClim versión 2.0. El área bajo la curva del modelo generado tuvo un valor de 0.98 , lo cual indica que es un modelo con buena confiabilidad. Los resultados mostraron áreas de idoneidad de hábitat en la costa sur de Alaska, costa este y oeste de Canadá, centro de Estados Unidos, Colombia, Ecuador, Perú, sur de Chile, sur de Brasil, costa este de Argentina y Uruguay. Esta información puede ser útil en los programas de vigilancia entomológica para el monitoreo de C. melanura en países como Estados Unidos y Canadá donde actualmente esta especie está presente.
\end{abstract}

Palabras-clave: Biomas; Culicoidea; modelo de distribución; vector.

\section{Distribuição potencial de Culiseta melanura (Coquillett) (Diptera: Culicidae) na América}

Resumo. Culiseta melanura é um mosquito importante devido ao seu papel no ciclo de transmissão do vírus da encefalite equina oriental. Neste estudo determinamos a distribuição potencial de C. melanura nas Américas e a importância das variáveis bioclimáticas na formação dessa distribuição. Foi utilizado um modelo de entropia máxima (MaxEnt, versão 3.4.1) para representar a potencial distribuição de C. melanura. Foi utilizado um total de 80 registros geográficos obtidos do GBIF e literatura relevante. Os dados climáticos foram obtidos a partir de 19 camadas climáticas disponíveis na base de dados WorldClim versão 2.0. A área sob a curva do modelo gerado teve um valor de 0,98, indicando que se trata de um modelo com boa confiabilidade. Os resultados mostraram áreas de aptidão de habitat na costa sul do Alasca, costa leste e oeste do Canadá, centro dos Estados Unidos, Colômbia, Equador, Peru, sul do Chile, sul do Brasil, costa leste da Argentina e Uruguai. Esta informação pode ser útil em programas de vigilância entomológica para a monitoramento da C. melanura em países como os Estados Unidos e o Canadá, onde esta espécie está presente atualmente.

Palavras-chave: Biomas; Culicoidea; padrão de distribuição; vetor.

El conocimiento de la distribución de las especies es fundamental en campos como la ecología, biogeografía, conservación y salud pública (CHAVE et al., 2002; GRAHAM y HIJMANS, 2006). En los últimos años se ha observado un incremento en el uso de los modelos de distribución de especies (MEROW et al., 2013; HERNÁNDEZ-SILVA et al., 2020), los cuales para su elaboración se basan en las localidades de recolecta y observación georreferenciadas, los requerimientos ecológicos y la relación de las especies con las variables ambientales que inciden en su área de distribución conocida (ELITH et al., 2006; PHILLIPS et al., 2017).

Los modelos de distribución son útiles ya que permiten evaluar el efecto que podría tener algún cambio en el ambiente sobre la distribución de una especie en particular (ANDERSON et al., 2002; FRANKLIN, 2009), así permite proyectar a futuro y en relación al cambio climático la distribución de especies de importancia en áreas como la salud pública (LIU et al.,

\section{Editado por:}

Mateus Aparecido Clemente

\section{Histórico Editoral:}

Recebido em: 29.03.2021

Aceito em: 24.05.2021

Publicado em: 02.06.2021
2019). Por ejemplo, algunas especies de mosquitos dentro de la familia Culicidae están involucradas en la trasmisión de patógenos que afectan al hombre y a los animales domésticos (HARBACH, 2021), por lo que el conocimiento de su distribución es importante para establecer programas de control y manejo en áreas o regiones de interés para salud pública (KHAN et al., 2020; ESPINOSA-VÉLEZ et al., 2020).

Culiseta melanura (Coquillett) (Diptera: Culicidae) es un mosquito importante debido a su papel en el ciclo de transmisión del virus de la Encefalitis Equina del Este y potencialmente del virus del Nilo Occidental (CUPP et al., 2003; MOLAEI y ANDREADIS, 2006). Esta especie es señalada con una biología inusual debido a que pasa el invierno en estado larval; mientras que la mayoría de las especies de mosquitos pasan el invierno como adultos o huevos. Culiseta melanura se distribuye en áreas pantanosas de tierras bajas desde el sur de Quebec, Canadá a través de Maine y el área de los Grandes Lagos hasta el sur de Florida y el

\section{Autor Correspondente:}

Rahuel J. Chan-Chablé

rahuel_jere_1990@hotmail.com

Agência(s) de Fomentos:

Consejo Nacional de Ciencia y Tecnología (CONACYT) de México 
Este de Texas, Estados Unidos (CRANS y MAHMOOD, 1998). Sus larvas se han colectado en el estado de Nuevo León, noreste de México (ORTEGA-MORALES et al., 2009). Algunos autores mencionan que se encuentra en el Caribe (HORSFALL, 1955; BUCKNER et al., 2012). Este estudio tuvo como objetivo estimar la distribución potencial de $C$. melanura en el continente americano y determinar la importancia de las variables bioclimáticas en la conformación de dicha distribución.

Se descargaron de la base de datos GBIF (Global Biodiversity Information Facility) registros geográficos de $C$. melanuara (GBIF, 2021). Los registros obtenidos pasaron por un proceso de depuración, dándole prioridad a los datos publicados por taxónomos expertos en el grupo, hasta obtener aquellos de mayor confiabilidad. Adicionalmente, se realizó una revisión bibliográfica para la búsqueda de registros geográficos en las siguientes bases de datos: Google Scholar, PubMed Health, SciELO (Scientific Electronic Library Online) y Web of Science (Thompson Reuters). La búsqueda se realizó con la introducción de palabras clave como: C. melanura, C. melanura distribución, registro C. melanura, C. melanura Canadá, C. melanura México, y C. melanura Estados Unidos.

Los datos de clima se obtuvieron de 19 capas climáticas disponibles en la base de datos WorldClim versión 2.0 (FICK y HIJMANS, 2017). Para la selección de variables ambientales, se agregaron 10,000 puntos de fondo al polígono de área de distribución de C. melanura (CRANS y MAHMOOD, 1998), generada a partir de los datos de registro obtenidos de nuestra búsqueda en bases de datos y literatura pertinente. A dichos puntos se agregó información de las 19 variables ambientales del clima actual. Con la información generada se realizó un análisis de correlación bivariada para reducir la multicolinealidad entre las variables de entrada; para las variables que se encontraban altamente correlacionadas $(r>$ $0,75)$ se escogió la variable que presentó mayor variación o la que representó mayor significado biológico para la especie (CARDOZA-MARTÍNEZ et al., 2019).

Para representar la distribución potencial de C. melanura se utilizó un modelo de máxima entropía (MaxEnt, versión 3.4.1) (PHILLIPS et al., 2017). Se eligió el modelo MaxEnt porque utiliza datos de registros de presencia, es decir, ausencias seleccionadas aleatoriamente de áreas que han sido accesibles para la especie (CARDOZA-MARTíNEZ et al., 2019). MaxEnt tiene un mejor desempeño que los modelos de ausencia de presencia; además, este método de modelación puede manejar pequeñas muestras de los datos de presencia de una especie, a partir de cinco registros geográficos (CARDOZA-MARTíNEZ et al., 2019).

MaxEnt predice la idoneidad de hábitat en función de las variables ambientales y los datos de ocurrencia de las especies. Esta idoneidad de hábitat está representada por una escala que va desde 0 (idoneidad baja) a 1 (fitness alta) (BUCKLEY et al., 2008; CARDOZA-MARTÍNEZ et al., 2019). Para reducir la complejidad del modelo es necesario su respectiva evaluación y calibración (CARDOZA-MARTíNEZ et al., 2019), considerando las opciones de: a) Área accesible (background o área M), b) los tipos de variables que MaxEnt construye (features), y c) el tipo de salida del modelo (raw, acumulativa o logística), ya que estas consideraciones afectan las inferencias a realizar (MEROW et al., 2013; CARDOZA-MARTíNEZ et al., 2019). En este estudio, el método de calibración y evaluación para el modelado de C. melanura se llevó a cabo utilizando la librería "ENMeval" (CARDOZA-MARTÍNEZ et al., 2019) en el software estadístico R versión 3.5.2 (R Development Team, 2021).

Utilizando el software MaxEnt la información obtenida del modelo calibrado se proyectó hacia el continente americano considerando siete variables ambientales seleccionadas con base en los valores del análisis de correlación bivariada y tomando en cuenta la biología de C. melanura. Para obtener un modelo de nicho ecológico para C. melanura, representado geográficamente como un mapa de idoneidad de hábitat, basado en las condiciones climáticas actuales se realizaron 15 repeticiones. Se consideró para la elaboración del modelo un total de 80 registros geográficos y las siete variables climáticas identificadas anteriormente.

Los modelos obtenidos con Maxent, fueron evaluados estadísticamente analizando el área bajo la curva (AUC: Area Under the Curve). AUC varía de 0 a 1, entre más cercano sea a 1 el valor quiere decir que el modelo tiene buen desempeño, mientras que valores cercanos o por debajo de 0,5 indican que el modelo no es más informativo que lo obtenido por azar (ORTíZ-YUSTY et al., 2014). Para obtener el mapa de distribución potencial de $C$. melanura se eligió el modelo con el mejor desempeño evaluado; este modelo fue convertido en un mapa de ausencia/presencia eliminando las áreas de ausencia a través de la reclasificación y estandarización de los valores usando el software ArcGis v. 10.5 (ESRI, 2011).

Para determinar la importancia de cada variable bioclimática en la conformación de la distribución geográfica obtenida de C. melanura, cada una de las siete variables fue evaluado de acuerdo a su importancia relativa al agregar el porcentaje de contribución (PC) y la importancia de la permutación (IP) evaluada por MaxEnt (CARDOZA-MARTÍNEZ et al., 2019). Adicionalmente, se realizaron análisis de Jackknife, en los que se ajustan los modelos con una sola variable a la vez y se midió el ajuste respecto al modelo completo con todas las variables elegidas (ORTíZ- YUSTY et al., 2014). Seguidamente, se ajustaron los modelos excluyendo una variable determinada y se compararon nuevamente con el modelo completo. Con este procedimiento se puede identificar las variables que más información aportan al modelo por sí solas y las variables que más información poseen.

Como resultados de la búsqueda en la base de datos GBIF se descargaron 83 registros geográficos para C. melanura, de los cuales se utilizó sólo 16, aquellos de mayor confiabilidad debido a que señalan el nombre del taxónomo quien identifico y están asociados a artículos científicos ya publicados, para generar el modelo de distribución. Por otra parte, se recuperó 74 datos de registros geográficos en 25 artículos de investigación sobre distribución de C. melanura, siendo un total de 80 registros geográficos utilizados en este estudio.

Con base en los valores del análisis de correlación bivariada y tomando en cuenta la biología de C. melanura se seleccionaron para el modelado las siguientes siete variables bioclimáticas: intervalo diurno promedio (bio2), temperatura mínima del mes más frío (bio6), temperatura media del trimestre más húmedo (bio8), precipitación anual (bio12), precipitación del mes más seco (bio14), estacionalidad de la precipitación (bio15), precipitación del trimestre más cálido (bio18).

El área bajo la curva del modelo generado con MaxEnt tuvo un valor de 0.98, lo cual indicó que es un modelo con buena confiabilidad. Las dos variables climáticas que mayor aportaron al modelo pertenecen a datos de precipitación; la estacionalidad de la precipitación (bio15) fue la variable que aportó mayor información al modelo al usarse individualmente, por lo tanto parece tener la información más útil por sí misma. La precipitación del trimestre más cálido (bio18) fue la variable ambiental que disminuye más la ganancia cuando se omite, lo que indica que posee la mayor cantidad de información que no está presente en las otras variables. Por otra parte, la temperatura mínima del mes más frío (bio6) y la precipitación del mes más seco (bio14) fueron las variables que menos aportaron al modelo de distribución de $C$. melanura con valores de $2.2 \%$ y $2.0 \%$ respectivamente (Tabla 1 ).

Finalmente, el modelo de distribución potencial de $C$. melanura en América mostró áreas de idoneidad de hábitat en la costa sur de Alaska, costa este y oeste de Canadá, centro de Estados Unidos (sureste del estado de Colorado), Colombia, Ecuador, 
Perú, sur de Chile, sur de Brasil, costa este de Argentina y Uruguay (Figura 1). Si bien existen barreras climáticas y geográficas como el desierto Chihuahuense y la cordillera de los Andes que pudieran impedir la introducción de esta especie a éstos países, no se debe descartar dicha posibilidad ya que la actividad antropogénica como el comercio pudiera hacer lo propio como en el caso de otras especies (Aedes aegypti, Ae. albopictus). Esta información será útil en los programas de vigilancia entomológica para el monitoreo de C. melanura en países como Estados Unidos y Canadá donde actualmente esta especie está presente.

Tabla 1. Porcentaje de contribución e importancia de permutación de las variables climáticas en la distribución de Culiseta melanura en el continente americano.

\begin{tabular}{lcc}
\hline Variable* & \% contribución & $\begin{array}{c}\text { Importancia de } \\
\text { permutación }\end{array}$ \\
\hline bio15 & 43.1 & 16.3 \\
\hline bio18 & 19.3 & 23.0 \\
\hline bio8 & 16.2 & 16.7 \\
\hline bio2 & 11.1 & 0.2 \\
\hline bio12 & 6.2 & 25.1 \\
\hline bio6 & 2.2 & 5.6 \\
\hline bio14 & 2.0 & 13.2 \\
\hline
\end{tabular}

*bio15 = estacionalidad de la precipitación, bio18 = precipitación del trimestre más cálido, bio 8 = temperatura media del trimestre más húmedo, bio2 = intervalo diurno promedio, bio12 = precipitación anual, bio6 = temperatura mínima del mes más frío, bio14 = precipitación del mes más seco.

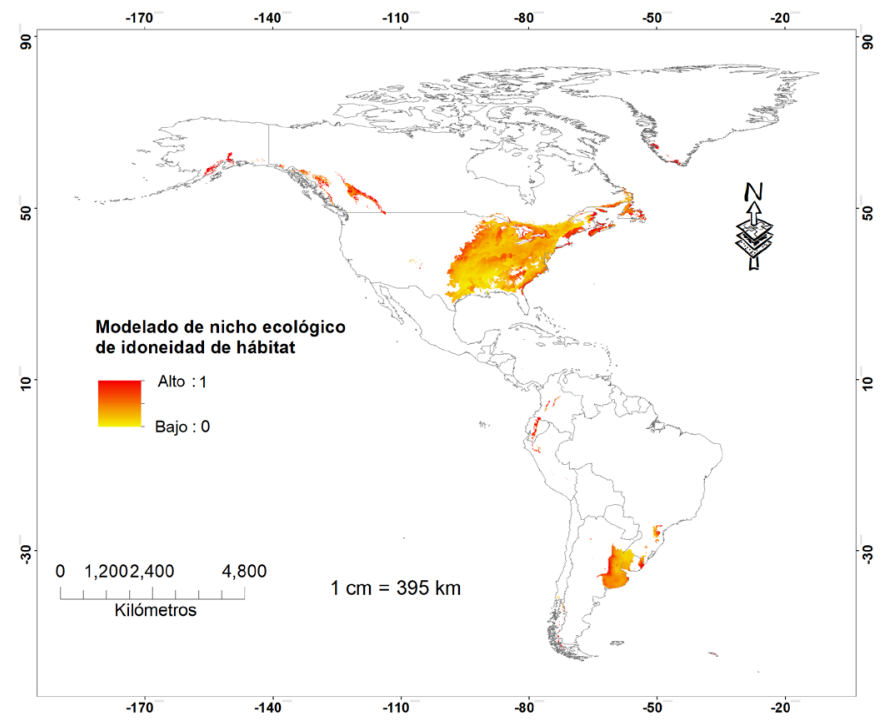

Figura 1. Modelo de distribución potencial de Culiseta melanura en America.

\section{Agradecimientos}

Agradecemos al Dr. Jorge L. Becerra por compartir sus conocimientos durante la impartición del curso diplomado, al MSP. Rafael Vázquez Marroquín por su apoyo con el equipo de cómputo para el procesamiento de los datos y proyección del modelo. Al Consejo Nacional de Ciencia y Tecnología (CONACYT) de México por la beca otorgado a Rahuel J. Chan-Chable.

\section{Referencias}

ANDERSON, R. P.; PETERSON, A. T.; GÓMEZ-LAVERDE, M. Using niche based GIS modeling to test geographic predictions of competitive exclusion and competitive release in South
American pocket mice. Oikos, v. 98, n. 1, p. 3-16, 2002. DOI: https://doi.org/10.1034/j.1600-0706.2002.t01-1-980116.x

BUCKNER, E.; SHOWMAN, A.; CONNELLY, C. R. Featured Creatures: common name: black-tailed mosquito scientific name: Culiseta melanura (Coquillett) (Insecta: Diptera: Culicidae). 2012. University of Florida. Disponible en: <http:// entnemdept.ufl.edu/creatures/aquatic/Culiseta_melanura. htm>. Consultado el: 27 Mar. 2021.

BUCKLEY, L. B.; RODDA, G. H.; JETZ, W. Thermal and energetic constraints on ectotherm abundance: $A$ global test using lizards. Ecology, v. 89, n. 1, p. 48-55, 2008. DOI: https://doi.org/10.1890/07-0845.1

CARDOZA-MARTÍNEZ, G. F.; BECERRA-LÓPEZ, J. L.; ESPARZAESTRADA, C. E.; ESTRADA-RODRÍGUEZ, J. L.; CZAJA, A.; EHSAN, M.; ROMERO-MÉNDEZ, U. Shifts in climatic niche occupation in Astrophytum coahuilense ( $\mathrm{H}$. Möller) Kayser and its potential distribution in Mexico. Sustainability, v. 11, n. 4, p. 1138, 2019. DOI: https://doi.org/10.3390/su11041138

CHAVE, J.; MULLER-LANDAU, H. C.; LEVIN, S. A. Comparing classical community models: theoretical consequences for patterns of diversity. The American Naturalist, v. 159, n. 1, p. 1-23, 2002. DOI: https://doi.org/ 10.1086/324112

CRANS, W. J.; MAHMOOD, F. Evidence for a bi-voltine life cycle in Culiseta melanura. 1998. Northeastern Mosquito Control Association. Disponible en: <http://www.nmca.org/Nmca98-8. htm>. Consultado el: 28 Mar. 2021.

CUPP, E. W.; KLINGLER, K.; HASSAN, H. K.; VLGUERS, L. M.; UNNASCH, T. R. Transmission of eastern equine encephalomyelitis virus in central Alabama. American Journal of Tropical Medicine and Hygiene, v. 68, n. 4, p. 495-500, 2003.

ELITH, J.; GRAHAM, C. H.; ANDERSON, R. P.; DUDÍK, M.; FERRIER, S.; GUISAN, A.; HIJMANS, R. J.; HUETTMANN, F.; LEATHWICK, J. R.; LEHMANN, A.; LI, J.; LOHMANN, L. G.; LOISELLE, B. A.; MANION, G.; MORITZ, C.; NAKAMURA, M.; NAKAZAWA, Y.; OVERTON, J. M. M.; PETERSON, A. T.; PHILLIPS, S. J.; RICHARDSON, K.; SCACHETTIPEREIRA, R.; SCHAPIRE, R. E.; SOBERÓN, J.; WILLIAMS, S.; WISZ, M. S.; ZIMMERMANN, N. E. Novel methods improve prediction of species' distributions from occurrence data. Ecography, v. 29, n. 2, p. 129-151, 2006. DOI: https://doi.org/10.1111/j.2006.09067590.04596.x

ESPINOSA-VÉLEZ, Y.; ALTAMIRANDA-SAAVEDRA, M.; CORREA, M. M. Potential distribution of main malaria vector species in the endemic Colombian Pacific region. Tropical Medicine \& International Health, v. 25, n. 7, p. 861-873, 2020. DOI: https://doi.org/10.1111/tmi.13399

ESRI. ArcGIS Desktop: Release 10. 2011. Environmental Systems Research Institute, Redlands, CA. Disponible en: <https://desktop.arcgis.com/en/>. Consultado el: 30 Mar. 2021

FICK, S. E.; HIJMANS, R. J. Worldclim 2: New 1-km spatial resolution climate surfaces for global land areas. International Journal of Climatology, v. 37, n. 12, p. 4302-4315, 2017. DOI: 
https://doi.org/10.1002/joc.5086

FRANKLIN, J. (Ed.). Mapping species distributions: spatial inference and prediction. Cambridge: Cambridge University Press, 2009. 319p.

GBIF (Global Biodiversity Information Facility). Occurrence of Culiseta melanura. 2021. Disponible en: <https://www.gbif. org/occurrence/search?q=Culiseta\%20melanura>. Consultado el: 21 Ene. 2021.

GRAHAM, C. H.; HIJMANS, R. J. A comparison of methods for mapping species ranges and species richness. Global Ecology and biogeography, v. 15, n. 6, p. 578-587, 2006. DOI: https://doi.org/10.1111/j.1466-8238.2006.00257.x

HARBACH, R. E. Mosquito taxonomic inventory. 2021. Disponible en: <http://mosquito-taxonomic-inventory.info/ simpletaxonomy/term/6047>. Consultado el: 28 Mar. 2021.

HERNÁNDEZ-SILVA， N.; HERNÁNDEZ-RUIZ， J.; GONZALEZELIZONDO, M. S.; FIGUEROA-BRITO, R.; VILLARUANO, N. Distribución potencial de dos subespecies de Montanoa tomentosa (Asteracerae) en México. Revista de Biología Tropical, v. 68, n. 4, p. 1095-1106, 2020. DOI: https://doi.org/10.15517/rbt.v68i4.38624

HORSFALL, W. (Ed.). Mosquitoes, their bionomics and relation to disease. New York: The Ronald Press Company, 1955. 723p.

KHAN, S. U.; OGDEN, N. H.; FAZIL, A. A.; GACHON, P. H.; DUEYMES, G. U.; GREER, A. L.; NG, V. Current and Projected Distributions of Aedes aegypti and Ae. albopictus in Canada and the US. Environmental health perspectives, v. 128, n. 5, p. 057007, 2020. DOI: https://doi.org/10.1289/EHP5899

LIU, B.; GAO, X.; MA, J.; JIAO, Z.; XIAO, J.; HAYAT, M. A.; WANG, $H$. Modeling the present and future distribution of arbovirus vectors Aedes aegypti and Aedes albopictus under climate change scenarios in Mainland China. Science of
The Total Environment, v. 664, p. 203-214, 2019. DOI: https://doi.org/10.1016/j.scitotenv.2019.01.301

MEROW, C.; SMITH, M. J.; SILANDER, Jr. J. A. A practical guide to MaxEnt for modeling species' distributions: what it does, and why inputs and settings matter. Ecography, v. 36, n. 10, p. 1058-1069, 2013. DOI: https://doi.org/10.1111/j.16000587.2013.07872.x

MOLAEI, G.; ANDREADIS, T. G. Identification of avian- and mammalian-derived bloodmeals in Aedes vexans and Culiseta melanura (Diptera: Culicidae) and its implication for West Nile virus transmission in Connecticut, USA. Journal of Medical Entomology, v. 43, n. 5, p. 1088-1093, 2006. DOI: https://doi. org/10.1603/0022-2585(2006)43[1088:IOAAMB]2.0.CO;2

ORTEGA-MORALES, A. I.; ELIZONDO-QUIROGA, A.; GONZÁLEZVILLARREAL, A.; SILLER-RODRÍGUEZ, Q. K.; REYES-VILLANUEVA F.; FERNÁNDEZ-SALAS, I. First record of Culiseta melanura in Mexico, with additional Mexican records for Aedes sollicitans. Journal of the American Mosquito Control Association, v. 25, n. 1, p. 100-102, 2009. DOI: https://doi.org/10.2987/08-5774.1

ORTÍZ-YUSTY, C.; RESTREPO, A.; PÁEZ, V. P. Distribución potencial de Podocnemis lewyana (Reptilia: Podocnemididae) y su posible fluctuación bajo escenarios de cambio climático global. Acta Biológica Colombiana, v. 19, n. 3, p. 471-481, 2014. DOI: https://doi.org/10.15446/abc.v19n3.40909

PHILLIPS, S. J.; DUDÍK, M.; SCHAPIRE, R. E. Maxent software for modeling species niches and distributions (Version 3.4.1). 2017. Biodiversity Informatics. Disponible en: <http:// biodiversityinformatics.amnh.org/open_source/maxent/>. Consultado el: 27 Ene. 2021.

R DEVELOPMENT CORE TEAM. R: A language and environment for statistical computing. 2020. R Foundation for Statistical Computing. Disponible en: <http://www.R-project.org>. Consultado el: 27 Ene. 2021. 